\title{
A Retrospective Study on Relation between Cognitive Performance and Lobar Perfusions of Brain in Alzheimer's Dementia using Single Photon Emission Computer Tomography
}

Balachandar Rakesh, Srikala Bharath*, Bhavani Shankara Bagepally, Jitender Saini, Shilpa Sadanand, Naveen Donthi, Palanimuthu Thangaraju Sivakumar, Sadanandavally Ratnaswamy Chandra and Mathew Varghese

National Institute of Mental Health and Neurosciences, Bangalore, Karnataka, India

"Corresponding author: Srikala Bharath, MD, FRC Psych, DCAP, National Institute of Mental Health And Neurosciences, Bangalore, Karnataka, India, Tel: 918026995271; E-mail: srikala.bharath@gmail.com

Rec date: Apr 02, 2014, Acc date: June 26, 2014, Pub date: July 03, 2014

Copyright: (c) 2014 Rakesh B, et al. This is an open-access article distributed under the terms of the Creative Commons Attribution License, which permits unrestricted use, distribution, and reproduction in any medium, provided the original author and source are credited.

\begin{abstract}
Background and objectives: Alzheimer's disease (AD) is a chronic neurodegenerative condition that progressively affects all cognitive domains. Patients with AD have been documented to have perfusion deficits. We aim to study the perfusion of lobes and its role in cognition among patients with $A D$.

Methodology: Clinical data including cognitive scores on Hindi Mental Status Examination (HMSE) of 16 right handed patients with $A D$ visiting geriatric clinic were recorded. Single Photon Emission Computer Tomography (SPECT) was acquired with standard protocol. Region of interest was applied at each of the frontal, temporal, parietal, occipital, cerebellar lobes to measure the regional perfusion.

Results: Patients with AD aged $73.5 \pm 8.5$ years (6 males) with mean HMSE scores $11.69 \pm 5.4$ formed the study group. Left temporal lobe perfusion scores emerged as significant $(p=0.04)$ predictor of cognitive scores in patients with $A D$. We observed a significant difference in perfusion between individual lobes within right hemisphere $(p=0.003)$ and left hemisphere $(p=0.009)$.

Conclusion: In this sample of patients with $A D$ we were able to demonstrate the differential pattern of brain perfusion within the intra-hemispheric lobes, possibly due to differential rate of degeneration. We were able to demonstrate a possible association between the left temporal lobe perfusion and cognitive scores in patients with AD.
\end{abstract}

\section{Introduction}

Alzheimer's disease $(\mathrm{AD})$ is a chronic neurodegenerative condition characterized by deficits in a broad range of neuropsychological domains namely attention, learning, memory, executive functioning, processing speed, and language [1]. Perfusion studies involving patients with $\mathrm{AD}$ have documented deficits predominantly in parietal and temporal lobes. As the disease progresses there is global regional cerebral blood flow deficits involving other lobes [2].

Single Photon Emission Computed Tomography (SPECT) is a minimally invasive imaging tool to study the perfusion pattern of brain using radio tracers. Further it is potentially useful in determining the regional perfusion, regional cerebral blood flow ( $\mathrm{rCBF}$ ) and regional cerebral metabolism.

Patients with $\mathrm{AD}$ have been demonstrated to have reduced $\mathrm{rCBF}$, cerebral metabolism in temporal and parietal regions of brain [2]. To our best knowledge there is only one reported study that has explored the relation between regional perfusion and cognitive deficits in patients with $\mathrm{AD}[3]$. We attempted to explore the relation between the $\mathrm{rCBF}$ and cognitive symptoms in patients with $\mathrm{AD}$ using SPECT.

\section{Methodology}

\section{Location}

This is a retrospective study conducted at Geriatric Clinic Services of National Institute of Mental Health \& Neurosciences (NIMHANS), Bangalore, India. This study is part of a larger ongoing research protocol of the clinic. This study received clearance from the institutional ethical clearance committee.

\section{Sample and patient assessment}

Clinical data of 16 subjects (6 males) who underwent SPECT as a part of clinical evaluation, were collected. A diagnosis of AD was made on the basis of International Classification of Disease-10 by two clinicians (one of them being a senior consultant) after detailed clinical evaluation. As a part of clinical protocol following assessments were carried out to evaluate patients with dementia - Hindi Mental Status Examination (HMSE), Everyday Activities Scale for India (EASI) [4] to evaluate the functional ability, Clinical Dementia Rating Scale (CDR) [5] for classifying the severity of Dementia, physical examination and relevant laboratory tests (such as thyroid functions tests, vitamin B12) to rule out secondary dementias. 
Citation: Rakesh B, Bharath S, Bagepally BS, Saini J, Sadanand S, et al. (2014) A Retrospective Study on Relation between Cognitive Performance and Lobar Perfusions of Brain in Alzheimer's Dementia using Single Photon Emission Computer Tomography . Brain Disord Ther 3: 135. doi:10.4172/2168-975X.1000135

Page 2 of 4

\section{Cognitive assessment}

Hindi Mental Status Examination a component of Cognitive Scale Battery (CSB) [6], similar to Mini Mental State examination is used for screening cognitive functions. Hindi Mental Status Examination consists of 22 questions and broadly covers Orientation, language (both comprehension and expressive speech), memory, concentration, attention and praxis. HMSE provides a single total comprehensive screening score for overall cognitive performance [7].

\section{SPECT imaging acquisition}

Patients were injected with $20 \mathrm{mCi}( \pm 2)$ of metastable nuclear isomer of technetium-99 tagged with ethylcysteinate dimer $(99 \mathrm{mTc}-$ ECD) under aseptic precautions. Patients were made to rest calmly in a low-lighted room for a period of 60 minutes after which they were shifted under SPECT gamma camera. SPECT images were acquired with SIEMENS symbia T6 machine, loaded with Low Energy High Resolution (LEHR) collimators. SPECT studies were carried out with a high-resolution collimator and a $128 \times 128$ matrix. There were 2 detectors that rotate $180^{\circ}$ degrees in a clockwise non circular orbital manner with step and shoot method. Images were acquired with a 15second per image- acquisition time, and a zoom of 1.45. Images were iteratively reconstructed. Reconstructed brain slices $(0.8 \mathrm{~cm}$ thickness) were then reoriented in the orbito-meatal line using the sagittal and axial views, and a set of axial, sagittal, and coronal sections were obtained using a 6 slice CT. SPECT image was acquired after consenting from the patient/the patient primary care-taker.

\section{Image analysis \& ROI}

Circular region of interest (ROI) was applied manually by the first author RB and confirmed by JS a consultant neuro-radiologist, on frontal, temporal, parietal, occipital and cerebellum lobes.

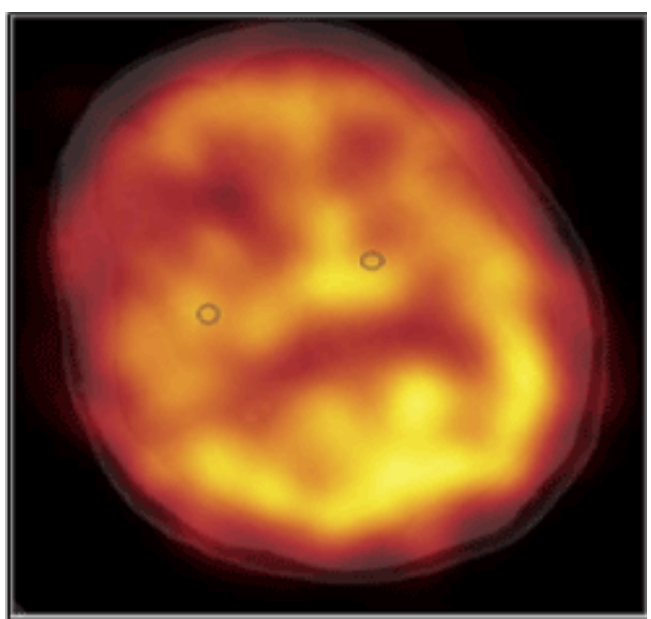

Figure 1: Showing an ROI on temporal lobe

The circular ROI measured 20 voxels in each of the lobes. ROI at frontal lobe was applied on frontal lobe 2 axial sections higher to the orbital level. Temporal lobe ROI was applied to the regions medial to the temporal horn of lateral ventricle. Parietal lobe ROI was applied on regions posterior to the central sulcus, 2 axial sections higher to the corpus callosum, occipital lobe ROI was applied on the regions 2 sections above the cerebellum. ROI for cerebellum was applied on the cerebellum at the level of middle cerebellar peduncle. ROI for left and right hemisphere lobes were applied separately. Total radiotracer counts for each of the ROI ( 20 voxels) were recorded (Figure 1 show a ROI on temporal lobe).

\section{Statistical model}

The tracer uptake data from all the ROI's followed normal distribution (tested using Shapiro-Wilk test). Perfusion counts of the lobes were standardized by dividing them with respective ipsilateral cerebellar perfusion counts (cerebral/ cerebellum) [8]. In order to detect the perfusion pattern in the lobes, Analysis of Variance (ANOVA) was performed with 4 lobes of same hemispheres individually. Post hoc Bonferroni test was performed to correction for multiple comparisons. We performed regression analysis to determine which of the lobar perfusion better predicted the HMSE scores after correcting for influences of age and gender effects.

\section{Results}

\section{Demographic and clinical details}

All 16 subjects were right handed. Mean CDR was 2.06 (2 subjects with $\mathrm{CDR}=1,11$ subjects with $\mathrm{CDR}=2$ and 3 subjects with $\mathrm{CDR}=3$ ). Mean age of the sample was $73.46 \pm 8.5$ years. EASI scores were not available for all the subjects (Table 1).

\begin{tabular}{|l|l|l|l|}
\hline Patient ID & EASI & HMSE & CDR \\
\hline 1 & 7 & 11 & 2 \\
\hline 2 & 9 & 11 & 2 \\
\hline 3 & 8 & 11 & 2 \\
\hline 4 & NA & 10 & 2 \\
\hline 5 & 7 & 23 & 1 \\
\hline 6 & 8 & 10 & 2 \\
\hline 7 & 8 & 13 & 2 \\
\hline 8 & NA & 11 & 2 \\
\hline 9 & 5 & 19 & 2 \\
\hline 10 & 7 & 12 & 2 \\
\hline 11 & NA & 8 & 3 \\
\hline 12 & NA & 13 & 2 \\
\hline 13 & 12 & 1 & 3 \\
\hline 14 & 10 & 8 & 3 \\
\hline 15 & 8 & 12 & 2 \\
\hline 16 & 6 & 22 & 1 \\
\hline
\end{tabular}

Table 1: Showing the clinical details, NA: not available, HMSE: Hindi Mental Scale Examination, EASI: Everyday Ability Scale for India, CDR: Clinical Dementia Rating 
Citation: Rakesh B, Bharath S, Bagepally BS, Saini J, Sadanand S, et al. (2014) A Retrospective Study on Relation between Cognitive Performance and Lobar Perfusions of Brain in Alzheimer's Dementia using Single Photon Emission Computer Tomography . Brain Disord Ther 3: 135. doi:10.4172/2168-975X.1000135

Page 3 of 4

\section{SPECT results}

Our study observed a significant difference in the standardized perfusion scores within lobes of right hemisphere $(\mathrm{p}=0.003)$ and left hemisphere $(\mathrm{p}=0.009)$ in patients with $\mathrm{AD}(\mathrm{PwAD})$, with lowest being parietal and temporal lobes and highest being occipital lobes (table 2). There was no significant difference between the perfusions of right and left hemisphere. Our study observed left temporal lobe perfusion was significantly $(\mathrm{p}<0.05$, with $\mathrm{R} 2=0.32)$ a better predictor of total HMSE scores (Figure 2).

\begin{tabular}{|c|c|c|c|c|}
\hline Lobes & Right (R) & Left (L) & $\begin{array}{l}\text { Significance } \\
\text { (p) }\end{array}$ & $\begin{array}{l}\text { Significance } \\
\text { B/W R \& } \\
\text { hemisphere }\end{array}$ \\
\hline Parietal & $0.67 \pm 0.08$ & $0.67 \pm 0.11$ & NS\# & \multirow[t]{5}{*}{ NS } \\
\hline Temporal & $0.72 \pm 0.24$ & $0.74 \pm 0.16$ & NS\# & \\
\hline Frontal & $0.83 \pm 0.16$ & $0.83 \pm 0.18$ & NS\# & \\
\hline Occipital & $0.92 \pm 0.15$ & $0.87 \pm 0.17$ & NS\# & \\
\hline $\begin{array}{l}\text { Significance } \\
\text { (P) }\end{array}$ & $P=0.003^{*}$ & $P=0.009^{*}$ & - & \\
\hline
\end{tabular}

Table 2: Showing lobar perfusion, NS\#: Not significant, ${ }^{*}$ : Significant

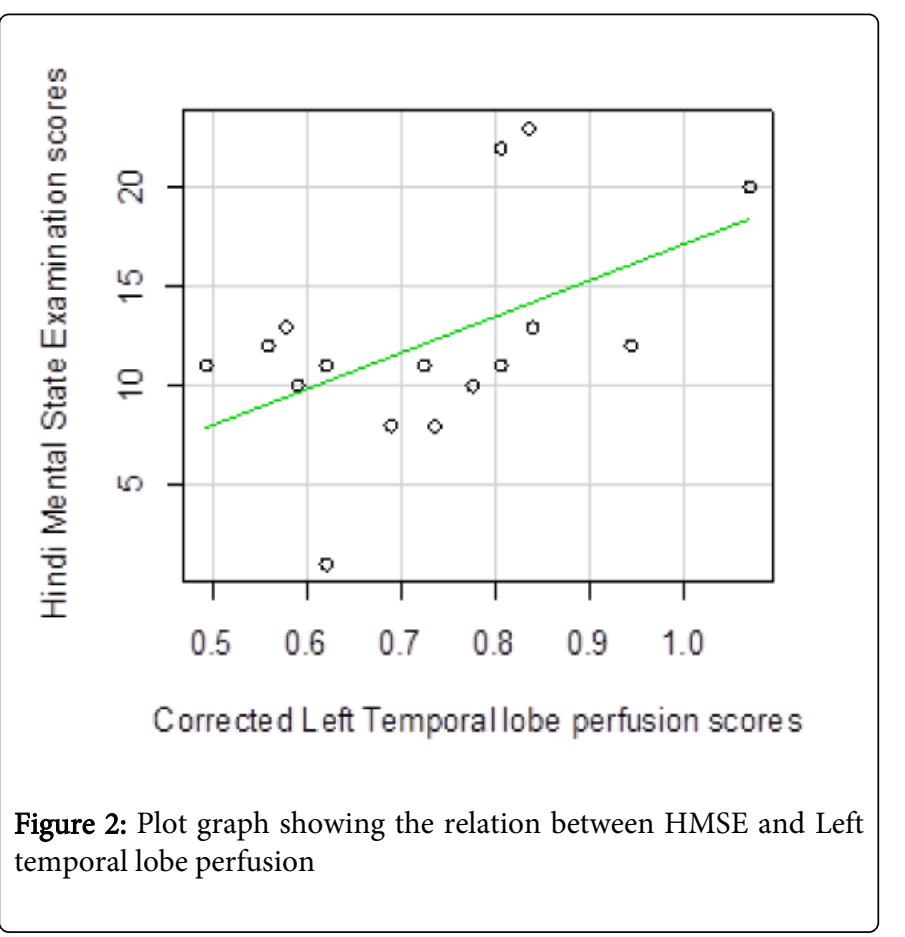

\section{Discussion}

Here in this study we tried to explore the possible relation between lobar perfusion and cognitive functions in PwAD using ECD-SPECT. We observed a positive association between left temporal lobe perfusion and cognitive scores (assessed by HMSE). Other notable findings of this study being non-uniform lobar perfusion in PwAD and relative lower temporo-parietal lobar perfusion.

Our findings of left temporal lobe perfusion being a better predictor of cognitive performance in $\mathrm{AD}$ has been reported in the past studies too [3]. Cognitive functions of Temporal lobe has been demonstrated in patients with temporal lesions. These functions include verbal memory, language and verbal attention [9]. HMSE is sensitive in assessing deficits involving cognitive functions lateralized to dominant hemisphere such as verbal memory, verbal language and verbal attention. Hence this could be the possible reason for our finding of left temporal lobe perfusion being positively correlated with cognitive performance. Better the perfusion higher were the scores on HMSE.

Structural imaging (MRI) and functional (positron emission computer tomography) [10] studies on patients with $\mathrm{AD}$ have demonstrated the asymmetrical neuro-degeneration predominantly involving temporo-parietal lobes [11]. Amyloid plaques and neurofibrillary tangles - the neuro-pathognomic aspects of $\mathrm{AD}$ are reported to be richly deposited in temporal lobes and correlated with the severity of $\mathrm{AD}$ [12]. Our finding of relative lower perfusion in temporo-parietal lobes in PwAD could possibly be due to degenerative process preferably involving these lobes. The fact that pathogenesis of $\mathrm{AD}$ involves temporal and parietal lobes in the early stages and occipital lobes are relatively spared even in the later stages of the disease [13] is reflected even in our study.

Recent studies have started to throw light on role of small vessel pathology being contributing to the cognitive symptoms in patients with Alzheimer disease [14,15]. Various vascular pathologies such as cholesterol metabolism pathways (clusterin) vascular risk factors (insulin, hypertension) have been reported to play role in the structural atrophy Alzheimer's disease [16]. The fact whether degenerative changes lead to secondary perfusion deficits or vice versa is still inconclusive. As regional hypoperfusion is associated with cognitive symptoms in patients with $\mathrm{AD}$, whether improving the perfusion can retard/reverse the deficits in patients is yet to be explored. These are the questions that can provide insights in management of patients with AD.

Limitations of this study include low sample size, cognitive performance being assessed with a screening tool. Future case-control studies with detailed cognitive assessment using standardized neuropsychological battery for individual domains/lobes would be necessary to exactly predict the relationship between lobar perfusion and specific cognitive deficits.

\section{Conclusion}

Patients with $\mathrm{AD}$ have relatively lower perfusion in temporal and parietal lobes. There exists a relation between regional cerebral perfusion and cognitive deficits in patients with $\mathrm{AD}$. Future studies exploring the causal effect relationship between cerebral perfusion and cognitive deficits would possibly provide novel insight towards understanding the neuro-biology of $\mathrm{AD}$.

\section{Conflict of interest}

There is no conflict of interest

\section{References}

1. Twamley EW, Ropacki SA, Bondi MW (2006) Neuropsychological and neuroimaging changes in preclinical Alzheimer's disease. J Int Neuropsychol Soc 12: 707-735.

2. Hirsch C, Bartenstein P, Minoshima S, Mosch D, Willoch F, et al. (1997) Reduction of regional cerebral blood flow and cognitive impairment in 
Citation: Rakesh B, Bharath S, Bagepally BS, Saini J, Sadanand S, et al. (2014) A Retrospective Study on Relation between Cognitive Performance and Lobar Perfusions of Brain in Alzheimer's Dementia using Single Photon Emission Computer Tomography . Brain Disord Ther 3: 135. doi:10.4172/2168-975X.1000135

Page 4 of 4

patients with Alzheimer's disease: evaluation of an observer-independent analytic approach. Dement Geriatr Cogn Disord 8: 98-104.

3. Montaldi D, Brooks DN, McColl JH, Wyper D, Patterson J, et al. (1990) Measurements of regional cerebral blood flow and cognitive performance in Alzheimer's disease. J Neurol Neurosurg Psychiatry 53: 33-38.

4. Fillenbaum GG, Chandra V, Ganguli M, Pandav R, Gilby JE, et al. (1999) Development of an activities of daily living scale to screen for dementia in an illiterate rural older population in India. Age Ageing 28: 161-168.

5. Morris JC (1993) The Clinical Dementia Rating (CDR): current version and scoring rules. Neurology 43: 2412-2414.

6. Ganguli M, Chandra V, Gilby JE, Ratcliff G, Sharma SD, et al. (1996) Cognitive test performance in a community-based nondemented elderly sample in rural India: the Indo-U.S. Cross-National Dementia Epidemiology Study. Int Psychogeriatr 8: 507-524.

7. Ganguli M, Ratcliff G, Chandra V, Sharma S, Gilby J, et al. (1995) A hindi version of the MMSE: The development of a cognitive screening instrument for a largely illiterate rural elderly population in india. International Journal of Geriatric Psychiatry 10: 367-377.

8. Burns A, Philpot MP, Costa DC, Ell PJ, Levy R (1989) The investigation of Alzheimer's disease with single photon emission tomography. J Neurol Neurosurg Psychiatry 52: 248-253.

9. Bell B, Lin JJ, Seidenberg M, Hermann B (2011) The neurobiology of cognitive disorders in temporal lobe epilepsy. Nat Rev Neurol 7: 154-164.
10. Nordberg A, Rinne JO, Kadir A, Långström B (2010) The use of PET in Alzheimer disease. Nat Rev Neurol 6: 78-87.

11. Dickerson BC, Feczko E, Augustinack JC, Pacheco J, Morris JC, et al (2009) Differential effects of aging and Alzheimer's disease on medial temporal lobe cortical thickness and surface area. Neurobiol Aging 30: 432-440.

12. Archer HA, Edison P, Brooks DJ, Barnes J, Frost C, et al. (2006) Amyloid load and cerebral atrophy in Alzheimer's disease: an 11C-PIB positron emission tomography study. Ann Neurol 60: 145-147.

13. Lehmann M, Crutch SJ, Ridgway GR, Ridha BH, Barnes J, et al. (2011) Cortical thickness and voxel-based morphometry in posterior cortical atrophy and typical Alzheimer's disease. Neurobiol Aging 32: 1466-1476.

14. Kalaria RN (2002) Small vessel disease and Alzheimer's dementia: pathological considerations. Cerebrovasc Dis 13 Suppl 2: 48-52.

15. Marchesi VT (2011) Alzheimer's dementia begins as a disease of small blood vessels, damaged by oxidative-induced inflammation and dysregulated amyloid metabolism: implications for early detection and therapy. FASEB J 25: 5-13.

16. Thambisetty M, Simmons A, Hye A, Campbell J, Westman E, et al. (2011) Plasma biomarkers of brain atrophy in Alzheimer's disease. PLoS One 6: e28527. 